\title{
Crack Inpainting using Modified Cell Growth in Damaged Grayscale Images
}

\author{
I.Muthulakshmi \\ Assistant Professor, \\ VV College of Engineering \\ Tisaiyanvilai-627 657
}

\author{
D. Gnanadurai, PhD. \\ Principal, \\ J.P College of Engineering \\ Tenkasi - 627852
}

\begin{abstract}
This paper discusses an inpainting scheme for gray scale images. The scheme uses modified cell growth technique by which the damaged pixels are identified and then reconstructed by the mean of selected undamaged neighbor pixels. The canny edge detector is employed in the proposed scheme for finding the damaged neighbors for reconstruction. Thereby, the proposed scheme able to achieve the best PSNR. It is experimentally found that the proposed scheme provide best PSNR compared with well known existing filters Wiener, Median, Frost and Lee.
\end{abstract}

\section{Keywords}

Crack detection, canny edge detection, pixel point detection, modified cell growth.

\section{INTRODUCTION}

Human vision system (HVS) is one of the main input sources for the humans. At the same time, HVS is having limitation with vision illusion. Hence, visual information processing using computer is being researched from last few decades. The process of receiving and analyzing visual information by the human species is referred to as sight, perception or understanding. Similarly, the process of receiving and analyzing visual information by digital computer is called digital image processing.

Inpainting also known as interpolation, refers to the application of algorithms to reconstruct images by damaged regions where the data is lost or corrupted. The goal is to create an image that looks 'normal', meaning the viewer does not suspect that the image has been manipulated. The detection step is done by first determining the sub-block size in pixels used in the projection histogram. The crack detection is easy since most of the work is done in the pre-processing stage. The intensities are changed to represent the pixels accordingly. Thus, the reconstruction of damaged pixels is done. Such methodologies are well suited, if the carks are in straight lines. If the cracks are not straight lines or undefined shapes, reconstruction is failed. Hence, an effective inpainting scheme is always at greater demand.

The process, though effective, is very sensitive to noise and noise is represented just as apparently as the actual cracks. Inpainting techniques are used to restore historical images that took damage through the time. This kind of inpainting

or restoration is made by specialists. Removing image interference or just unwanted parts of an image is another area inpainting techniques are used [1]. This paper discusses an algorithm that does the inpainting automatically, requiring little amount of user input. Canny edge detector based reconstruction process in proposed in the paper [2]. The first criterion is low error rate. It is important that edges occurring in images should not be missed and that there be no responses to non-edges. The second criterion is that the edge points be well localized. In other words, the distance between the edge pixels as found by the detector and the actual edge is to be at a minimum. A third criterion is to have only one response to a single edge. Based on these criteria, the canny edge detector first smoothes the image to eliminate and noise. It then finds the image gradient to highlight regions with high spatial derivatives. The algorithm then tracks along these regions and suppresses any pixel that is not at the maximum (nonmaximum suppression). Edges will occur at points the where the gradient is at a maximum. Therefore, all points not at a maximum should be suppressed.

In order to do this, the magnitude and direction of the gradient is computed at each pixel. Then for each pixel check if the magnitude of the gradient is greater at one pixel's distance away in either the positive or the negative direction perpendicular to the gradient. If the pixel is not greater than both, suppress it. The gradient array is now further reduced by hysteresis. Hysteresis is used to track along the remaining pixels that have not been suppressed.

Cell growth were originally conceived in the 1940s by Ulam and von Neumann. Their purpose was to provide a framework to investigate the behavior of complex, extended systems [3]. Mitchell carried out a very useful review of current research into uniform cell growth to solve the density and synchronization tasks in 1996 [4]. The density task is basically the attempt to change a random initial configuration of cell states all to the majority of the initial configuration. The synchronization task is the similar to the density task but the result is alternate states of 0 's and 1's in each Consecutive time step. Both these tasks are examples of cell growth performing emergent computation.

For a system to demonstrate emergent computation the system must use the actions of simple components using local information and communication to give rise to coordinated global information processing [5]. Emergent computation is what is described to occur in some natural systems such as hive minds or within our own brain, although we don't fully understand how these natural systems operate emergent computation can be simulated using some artificial systems. Cell growth are just one of these systems [6].

Chady proposed a feed-forward cell growth in [7]. It uses a cellular automaton with the cell neighbourhood connections implemented in a similar manner to that of a neural network. In the domain of image processing, Orovas and Austin have extensively researched cellular associative neural networks [8] [9]. The crack addition is performed based on the following 
method.

$$
Y(i, j)=\left\{\begin{array}{l}
S(i, j) ; 1-p \\
N(i, j) ; p
\end{array}\right.
$$

Where, $\mathrm{Y}(\mathrm{i}, \mathrm{j})$ is consider as a noisy (cracked) image pixel, $\mathrm{S}(\mathrm{i}, \mathrm{j})$ is the original image pixel, $\mathrm{N}(\mathrm{i}, \mathrm{j})$ is the Noisy pixels and $\mathrm{p}$ is the possibility of noise occurrence. Here, the noisy pixel is a very low intensity valued pixel with three levels. The noise density is consider by a three levels like as first level, second level and third level depending upon the presence of dark lines.

\section{PROPOSED METHOD}

The proposed system is illustrated in the figure 1. It removes the high density noise level with dark and very thick crack lines. The human body contains connectivity cells, the cells are automatically generated from the healthy cells energy for replacing damaged cells. So the damaged tissues are replaced by new generated healthy cells. In this concept at first can apply to the removal of crack image pixel from the gray scale image.

The damaged crack inpainting method having two major steps are,

1. Crack Detection(Target detection)

2. Crack Removal

\subsection{Crack Detection}

The proposed system uses three level of detection method. They are

1. Crack object detection

2. Crack object edge detection

3. Crack object point pixel detection.

The noise removal method is based on cell growth

\subsubsection{Crack Object Detection:}

There are many object detection methods in the crack detections, like as manual selection of crack object and some specified segmentation methods in different domains. Here, the detection of crack lines is based on the mean value of sub images. In the human body damaged cells are different from the healthy cells based on energy. So we can easily identify the damaged cells, likely the crack lines are identified based on the minimum mean value of the sub images.

The image is divided into many number of sub images and find out the mean value for individual sub images, finally find the minimum mean value fixed as a target.

The pixel values less than that of minimum mean value considered as a collection of dark pixel values. So we can easily find out the target crack lines in the Noisy image.

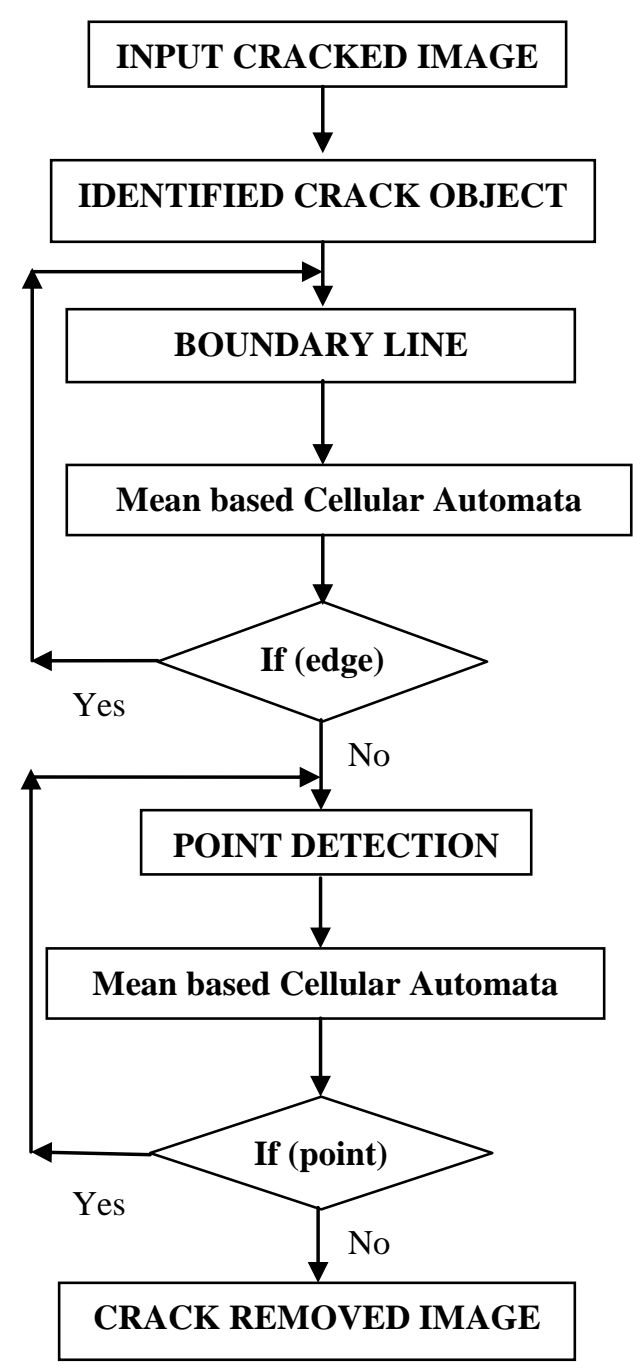

Fig 1: Flow Chart Diagram of Proposed method

Let's, consider the overall noisy image (512X512) is divided into $16 \mathrm{X} 16$ cell sized and find out the mean value for all 16X16 sized cells. The minimum mean value getting from that sized cells for the automatically identify the removal target of crack objects.

Mean_V(I)=Mean $(\operatorname{img}((\mathrm{M}+16) \mathrm{X}(\mathrm{N}+16)))$;

Mmean_value=min $($ Mean_V $)$;

Where, Mean_V (i) is the mean value $i^{\text {th }}$ cell from a noisy image. $I$ is the number of cells presents in the input image. Mmean_value is the minimum mean value from that all cell mean values.

$C(i, j)=\left\{\begin{array}{l}1 ; Y(i, j)<T \\ 0 ; \text { otherwise }\end{array}\right.$

Where, $\mathrm{C}(\mathrm{i}, \mathrm{j})$ is the binary image with crack object pixel. $\mathrm{T}$ is the minimum mean value (Mmean_value). If $Y(i, j)$ is less than the $T$, the pixel is identified crack pixel. Otherwise, the 
pixel considered a non targeted pixel. Now the removal target crack object find out from the noise image based on the Mmean_value.

The second step of proposed method is crack removal; it is based on edge line and pixel point detection from the target of crack area of input image.

\subsubsection{Crack object edge Detection:}

The crack edge detection is performed based on canny edge detection. The position of the crack edges are identified through the output of equation (2.3). The crack edge detection is continuously progressed, while detecting edges of crack objects. The number of edge detection and pixel replacing is called number of iteration.

$E(i, j)=\left\{\begin{array}{l}1 ; \text { if canny edge } \\ 0 ; \text { otherwise }\end{array}\right.$

Where, $E(i, j)$ is canny edge pixels based on the crack area of cracked image.

\subsubsection{Crack Point Pixel Detection:}

We can do the crack point pixel detection, after finishing edge detection based noise removal. Because the canny edge based noise removal have a chance to missing some crack pixel. So we need process with missed crack pixel. In this process is based the crack object detection. If any crack pixel in an area, it is detected with Mmean_value (2.2).

$P(i, j)=\left\{\begin{array}{l}1 ;\{Y 1(i, j)<T\} £ C \\ 0 ; \quad \text { otherwise }\end{array}\right.$

Where, $\mathrm{P}(\mathrm{i}, \mathrm{j})$ is the crack point pixel present in the removal of crack edges. Y1 is the output of crack edge detection and removal.

\subsection{Crack Removal:}

The crack removal is process with cell growth of modified cell growth [10]. The cell growth of modified cell growth is the function of healthy cell generation from the neighbor pixel collection of healthy cells [11].

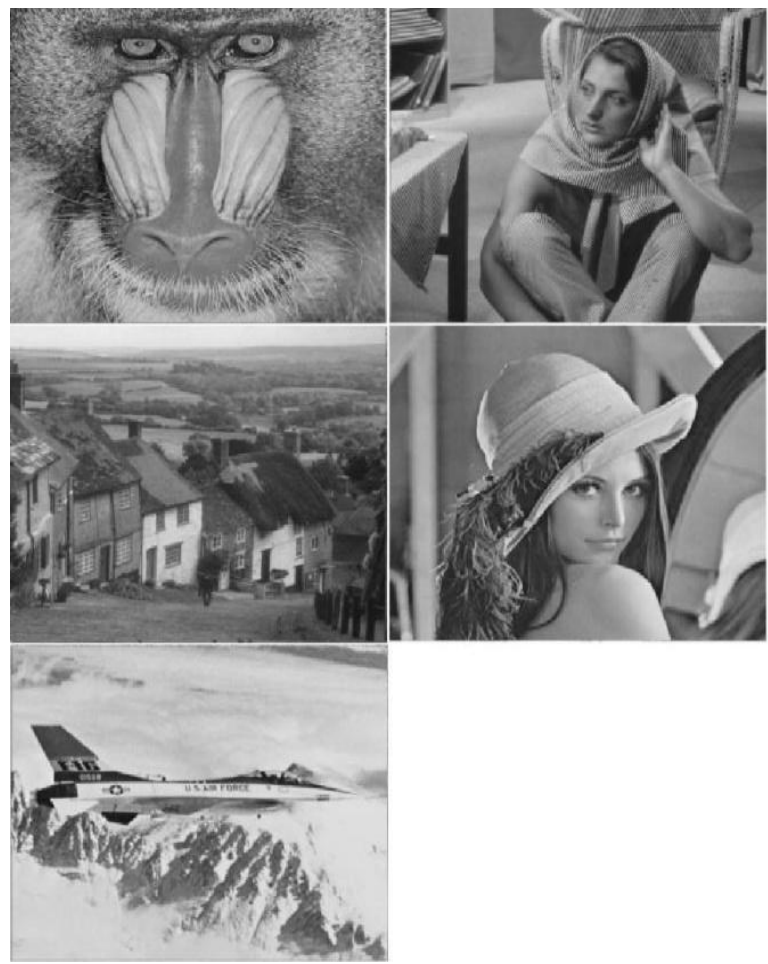

Fig 2: Test images of proposed scheme.

After even all iteration of edge and point pixel detection, the crack pixels are replaced by this propose crack removal method. If getting collection of edge pixel position, even all pixels can take individually process with window. Finally reconstructed pixel generated based on only neighbor of good pixels.

\begin{tabular}{|l|l|l|l|l|}
\hline 24 & 43 & 63 & 2 & 45 \\
\hline 54 & 24 & 65 & 2 & 54 \\
\hline 75 & 53 & 3 & 0 & 45 \\
\hline 64 & 53 & & 0 & 45 \\
\hline 57 & 65 & 1 & 0 & 45 \\
\hline
\end{tabular}

Fig 3: window $5 \times 5$ crack marked

\begin{tabular}{|l|l|l|l|l|}
\hline 0 & 0 & 0 & 1 & 0 \\
\hline 0 & 0 & 0 & 1 & 0 \\
\hline 0 & 0 & & 1 & 0 \\
\hline 0 & 0 & 1 & 1 & 0 \\
\hline 0 & 0 & 1 & 1 & 0 \\
\hline
\end{tabular}

Fig 4: window $5 \times 5$ binary image 


\begin{tabular}{|c|c|c|c|c|}
\hline 24 & 43 & 63 & 2 & 45 \\
\hline 54 & 24 & 65 & 2 & 54 \\
\hline 75 & 53 & \multirow{2}{*}{51} & 0 & 45 \\
\hline 64 & 53 & & 0 & 45 \\
\hline 57 & 65 & 1 & 0 & 45 \\
\hline
\end{tabular}

\section{Fig 5: Pixel replace for current pixel}

Consider red marked pixel position is crack area in $5 \mathrm{X} 5$ window. The current pixel intensity is 3

Mmean_value $=\operatorname{sum}(24,43,63,45,54,24,65,54,75,53$,

Mmean_value $=874 / 17$; $45,64,53,45,57,65,45) \quad(2.6)$

Mmean_value $=51.411$;

The mean value of the good neighboring pixels can replaced to current crack pixel. The number of iteration is based on the number detection \& removal.

\section{RESULT \& DISCUSSION}

The test images are taken in gray scale and the size is $512 \times 512$. The cracks are artificially created using filters. The three levels of darkest lines are placed for testing the proposed scheme. The individual test images and corresponding outputs are shown below.

Figure. 8 shows the cracked lines are processed with Lena image in the proposed method. So the corresponding images are displayed with cracked images. The proposed method results are discussed with the following parameters,

\subsection{Mean Square Error (MSE):}

In generally reconstructed images are discussed with MSE and PSNR value. MSE is calculated between input original noise free gray scale image and corresponding reconstructed image. This is the error calculation between input noise free image and crack removed image. So we need reduce the error by our proposed method, in output results can shown the reduced error than the error between crack image and input original image. It is most easily defined via the mean squared error (MSE). which for two $m \times n$ monochrome images $I$ and $K$. where one of the images is considered a noisy approximation of the other is defined as:

$$
M S E=\frac{1}{m n} \sum_{i=0}^{m-1} \sum_{j=0}^{n-1}[I(i, j)-K(i, j)]^{2}
$$

\subsection{Peak Signal to Noise Ratio (PSNR):}

The peak signal to noise ratio (PSNR) is defined as,

$\mathrm{PSNR}=10 \log _{10}\left(255^{2} / \mathrm{MSE}\right)$

PSNR is used for identify the signal power to the reconstructed image [12]. In this parameter is based on the MSE. Here, $255^{2}$ is the square of the peak value of the gray scale image intensity value and MSE is the mean square error. The reconstructed image quality is analyzed with above discussed parameters. The filters are wiener filter, median filter, Frost filter, Lee filter and Average filter. In this filters only giving best result for low density noisy image, the test images are high density noisy crack images. Hence, it could not perform well compared with the proposed scheme.

The Table 1 shows the output result analysis with comparisons of existing and proposed method. The various different existing noise removal filters results are compared with proposed method. Figure 8 shows the result of proposed method for crack level of Plan gray scale image. Figure 9 shows the result of proposed method for crack level of Baboon gray scale image and also Figure 11 shows the resultant images for the Hill Gray scale images.

The 512X512 size gray scale images are used for result comparison with existing and proposed method. Figure. 12 shown the crack is processed with our proposed algorithm and got the better result in grayscale image. In this crack is high density than compare to previous test image crack thickness. Some real time images are processed by our proposed algorithm of Crack Inpainting Using Modified Cell growth. That real time images are shown in figure 12 and other existing methods output results are shown in figure. 7 .

\section{CONCLUSION}

In this paper, an inpainting scheme based on cell growth and crack edge detector is proposed. It detects the damaged pixels through the minimum mean value of blocks considered as crack pixels. Then, the neighbors are calculated through the canny edge detector. Finally reconstruction is performed with the help of mean of undamaged neighborhood pixels with the concept of cell growth. It is experimentally found that the proposed scheme outperforms the existing well known filters wiener, median, frost and lee. 

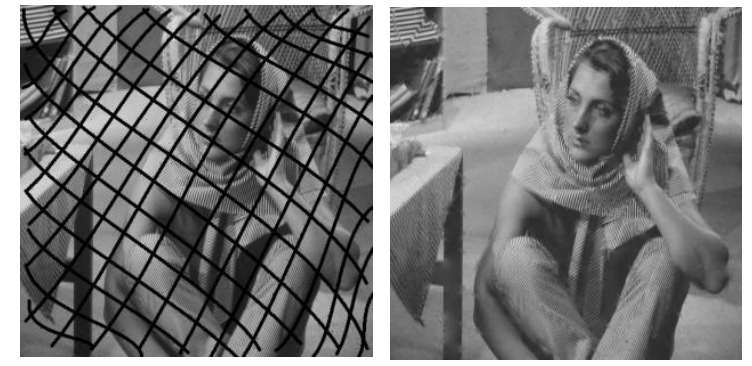

Fig. 6: Barbara grayscale outputs (512X512) PSNR: 34.8062
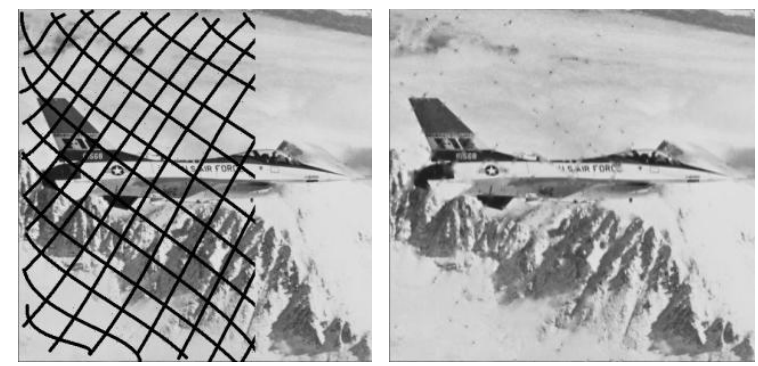

Fig 8: Plan Gray Scale image $\left(2^{\text {nd }}\right.$ level Crack \& Crack Removed) PSNR: 35.9081
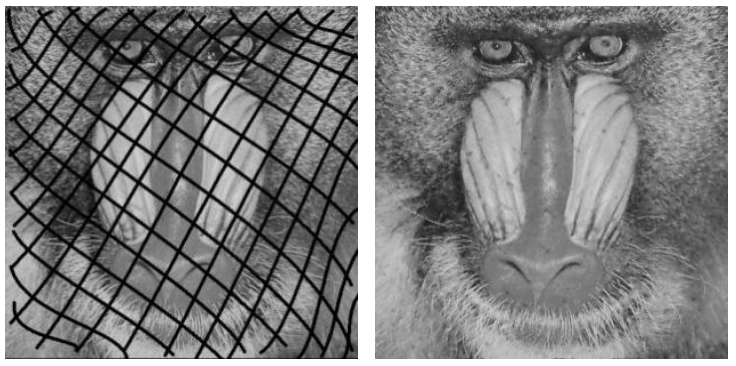

Fig 9: Baboon grayscale outputs (512X512)

PSNR: 32.3316

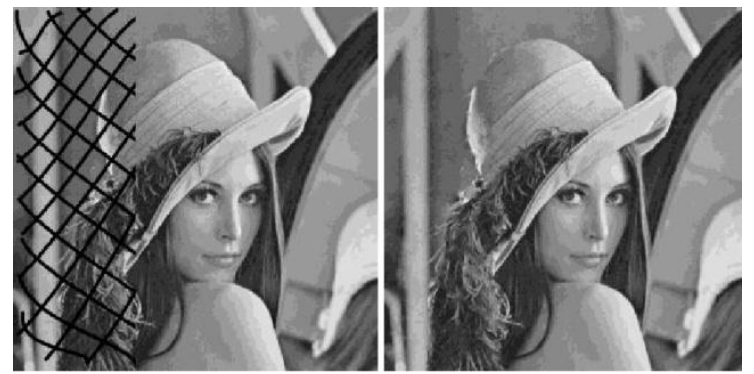

Fig 10: Lena Gray Scale image (Crack \& Crack Removed) PSNR: 35.3606

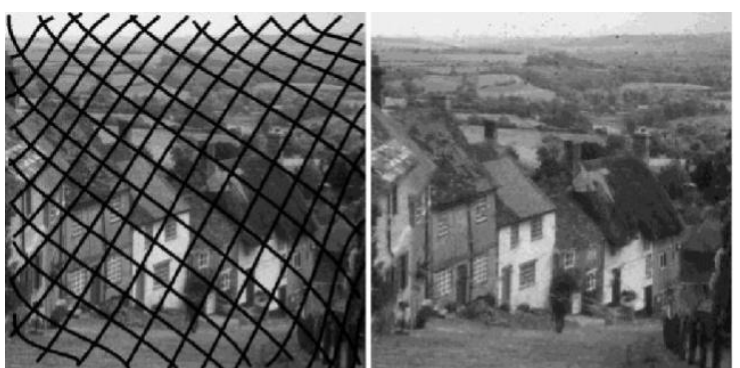

Fig 11: Hill grayscale outputs (512X512) PSNR: 34.5178
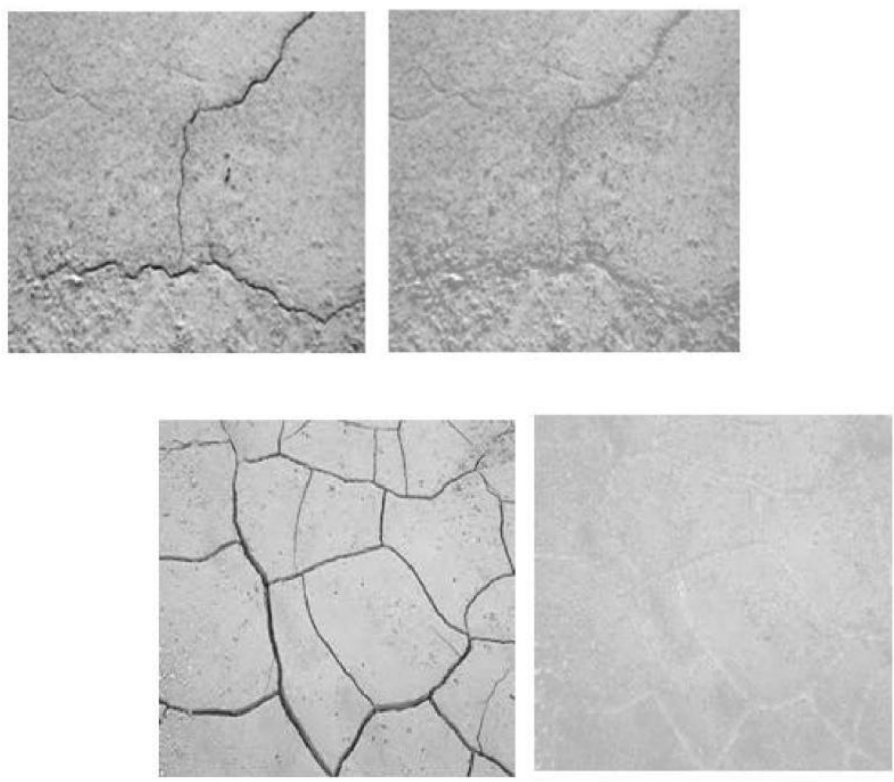

Fig 12: a) Real Time crack image b) crack removed image 
Table 1 Result Comparison of with Existing system.

\begin{tabular}{|c|c|c|c|c|c|c|c|c|}
\hline \multicolumn{2}{|l|}{ Method } & \multirow{2}{*}{$\begin{array}{l}\text { Noisy } \\
\text { PSNR }\end{array}$} & \multirow{2}{*}{$\begin{array}{l}\text { Wiener } \\
\text { PSNR }\end{array}$} & \multirow{2}{*}{$\begin{array}{l}\text { Median } \\
\text { PSNR }\end{array}$} & \multirow{2}{*}{$\begin{array}{l}\text { Frost } \\
\text { PSNR }\end{array}$} & \multirow{2}{*}{$\begin{array}{l}\text { Lee } \\
\text { PSNR }\end{array}$} & \multirow{2}{*}{$\begin{array}{l}\text { Average } \\
\text { PSNR }\end{array}$} & \multirow{2}{*}{$\begin{array}{l}\text { Proposed } \\
\text { PSNR }\end{array}$} \\
\hline Image & Crack & & & & & & & \\
\hline \multirow[t]{3}{*}{ Lena } & Level 1 & 29.815 & 29.40222 & 29.72776 & 28.04455 & 28.74287 & 28.65992 & 35.3606 \\
\hline & Level 2 & 31.5695 & 31.12424 & 31.32374 & 29.51014 & 30.3111 & 30.19179 & 37.1296 \\
\hline & Level 3 & 34.5133 & 33.76458 & 33.7742 & 31.67087 & 32.71888 & 32.51106 & 39.9476 \\
\hline \multirow[t]{3}{*}{ Hill } & Level 1 & 29.8333 & 29.10142 & 29.35144 & 27.85077 & 28.5546 & 28.48624 & 34.5178 \\
\hline & Level 2 & 31.5584 & 30.59723 & 30.7558 & 29.07818 & 29.92061 & 29.82274 & 36.1828 \\
\hline & Level 3 & 34.5037 & 32.74142 & 32.75207 & 30.83338 & 31.91537 & 31.76515 & 39.0662 \\
\hline \multirow[t]{3}{*}{ Barbara } & Level 1 & 29.8234 & 28.61804 & 28.87505 & 27.59326 & 28.13924 & 28.07659 & 34.8062 \\
\hline & Level 2 & 31.577 & 29.85154 & 30.04145 & 28.67336 & 29.27915 & 29.19273 & 36.4785 \\
\hline & Level 3 & 34.5041 & 31.42426 & 31.52279 & 29.91137 & 30.63458 & 30.51733 & 39.9576 \\
\hline \multirow[t]{3}{*}{ Plan } & Level 1 & 29.6969 & 29.13087 & 29.69819 & 27.60664 & 28.46667 & 28.45734 & 34.2581 \\
\hline & Level 2 & 31.4474 & 30.86187 & 31.27391 & 28.85451 & 29.93055 & 29.95685 & 35.9081 \\
\hline & Level 3 & 34.3821 & 33.39683 & 33.70498 & 30.67281 & 32.10946 & 32.16444 & 38.8571 \\
\hline \multirow[t]{3}{*}{ Baboon } & Level 1 & 29.5372 & 28.02046 & 28.20185 & 27.12745 & 27.63135 & 27.59664 & 32.3316 \\
\hline & Level 2 & 31.3016 & 28.92325 & 29.08162 & 27.91557 & 28.46826 & 28.42286 & 34.0866 \\
\hline & Level 3 & 34.182 & 30.26679 & 30.26769 & 29.07145 & 29.69314 & 29.63305 & 36.5509 \\
\hline
\end{tabular}
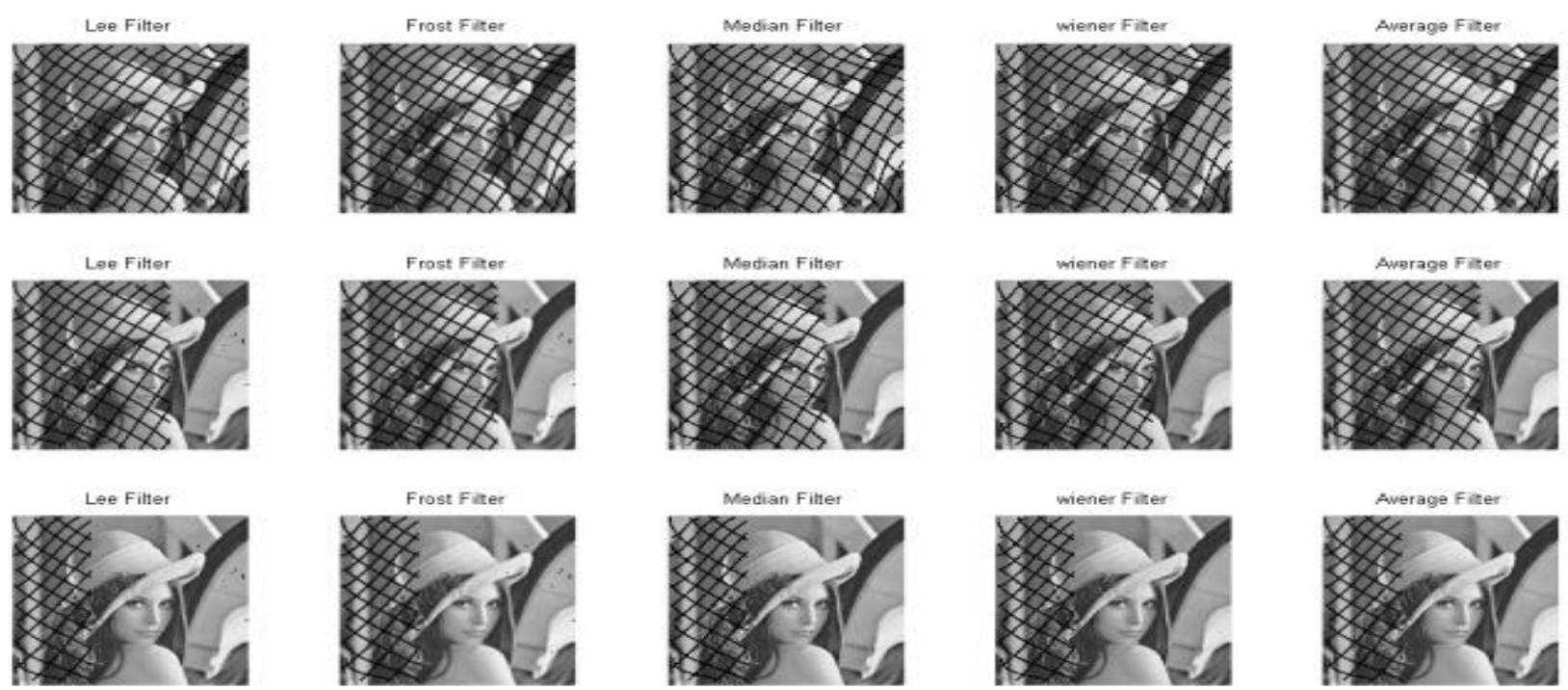

Fig 7: Different crack level filtered Lena grayscale outputs (512X512) by various filter 


\section{REFERENCES}

[1] Wen-Huang Cheng, Chun-Wei Hsieh, Sheng-Kai Lin, Chia-Wei Wang, and Ja-Ling Wu. Robust Algorithm for Exemplar-based Image Inpainting.

[2] Enayatifar R, Meybodi MR. Adaptive edge detection via image statistic features and hybrid model of fuzzy cellular automata and cellular learning automata. In: 2009 International Conference on Information and Multimedia Technology. 2009. p. 273-8.

[3] von Neumann, J. 1966. Theory of Self-Reproducing Automata. University of Illinois Press, Illinois. Edited and completed by A. W. Burks.

[4] M Mitchell, J.P. Crutchfield, R. Das 1996. Evolving Cellular Automata with Genetic Algorithms: A Review of Recent Work. Santa Fe Institute, Santa Fe, New Mexico \& IBM Watson Research Ctr, New York.

[5] Pradipta M, Chaudhuri PP. Fuzzy cellular automata for modeling pattern classi .er. IEICE Trans Inf Syst 2005;88:691.

[6] Hernandez G, Herrmann HJ. Cellular automata for elementary image enhancement. Graphic Models Image Process 1996;58:82-9.
[7] Chady, M. Poli, R. 1997. Evolution of Cellular Automatonbased Associative Memories. School of Computer Science, University of Birmingham, UK.

[8] Orovas, C. Austin, J 1997. Cellular Associative Neural Networks for Image Interpretation. Computer Science Department, University of York, UK

[9] Orovas, C. Austin, J 1997 Cellular Associative Symbolic Processing for Pattern Recognition. Computer Science Department, University of York, UK.

[10] Wolfram S. Theory and applications of cellular automata 1986.

[11] Sana Sadeghi,., Alireza Rezvanian, Ebrahim Kamrani. "An efficient method for impulse noise reduction from images using fuzzy cellular Automata", 2011.

[12] Rezvanian A, Rezvanian S, Khotanlou H. A new method to impulse noise reduction from medical images using cellular automata. In: Proceedings of the $17^{\text {th }}$ Iranian Conference on Electrical Engineering, ICEE, vol. 8. 2009. p. 53-8. 